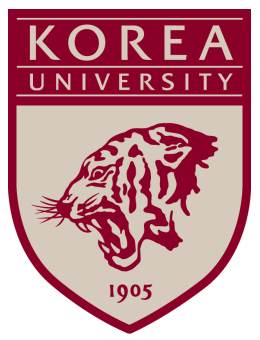

Discussion Paper Series

No. 1602

Jan 2016

\title{
Credit Market Frictions and Coessentiality of Money and Credit
}

\author{
Ohik Kwon and Manjong Lee
}

The Institute of Economic Research - Korea University

Anam-dong, Sungbuk-ku, Seoul, 136-701, South Korea, Tel: (82-2) 3290-1632, Fax: (82-2) 928-4948

Copyright (C) 2016 IER. 


\title{
Credit Market Frictions and Coessentiality of Money and Credit*
}

\author{
OHIK KWON \\ Department of Economics \\ Korea University \\ Seoul, Korea \\ k30113@korea.ac.kr
}

\author{
MANJONG LEE \\ Department of Economics \\ Korea University \\ Seoul, Korea \\ manjong@korea.ac.kr
}

\begin{abstract}
We explore how credit market frictions matter for the coessentiality of money and credit. There are high-productivity and low-productivity borrowers. Limited commitment can yield a one-for-one credit limit in accordance with a borrower's productivity. An adverse selection problem caused by asymmetric information, however, makes lenders impose the credit limit of a low-productivity borrower on a high-productivity borrower. If productivities differ sufficiently between borrowers, a high-productivity borrower is credit-constrained and is willing to hold money to compensate for the deficiency of her credit limit, but a low-productivity borrower is not. This eventually implies the coessentiality of money and credit in the sense that the use of both improves the allocation from a social welfare perspective.
\end{abstract}

Keywords: asymmetric information, adverse selection, cash, coessentiality, credit JEL classification: E41, E44, E50

${ }^{*}$ We thank Hyung Sun Choi, Jinill Kim, Randall Wright, and Seung Han Yoo for their helpful comments and suggestions. Financial support from the National Research Foundation of Korea Grant funded by the Korean Government (NRF-2014S1A3A2044238) is gratefully acknowledged. The usual disclaimer applies. 


\section{Introduction}

Credit as a payment instrument has become increasingly important and has attracted the keen attention of both economists and policymakers. In particular, monetary economists have tried to explore the coexistence of money and credit as means of exchange based on a microfounded monetary approach. ${ }^{1}$ Essentially, this strand of literature tried to provide in depth answers to the questions posed by Townsend (1989): "Can we find a physical environment in which currency-like objects play an essential role in implementing efficient allocation? Would these objects coexist with more ordinary, named credits?"

Recently, however, Gu, Mattesini and Wright (2015) show that in equilibrium where money and credit coexist, credit can have no social role. To put it differently, the coexistence of money and credit does not necessarily mean their coessentiality. (See also Lotz and Zhang 2015.) By coexistence we here mean that both money and credit are circulated as means of exchange, and by coessentiality we mean that the use of both improves the efficiency of allocations relative to an economy with money only or with credit only. This finding suggests that understanding the frictions that render money and credit coessential is as important as understanding the frictions that render them coexistent. Several recent works relate to this idea including Berentsen, Camera and Waller (2007), Gomis-Porqueras and Sanches (2013), and Araujo and Hu (2014). One of their shared features is that moderate inflation is necessary for coessentiality.

In this paper, rather than inflation as an ingredient of coessentiality, we investigate credit market frictions such as limited commitment and asymmetric information, which have returned to the spotlight in the recent global financial crisis. ${ }^{2}$ In order to do that,

\footnotetext{
${ }^{1}$ See, for instance, Shi (1996), Kocherlakota and Wallace (1998), Cavalcanti and Wallace (1999), Corbae and Ritter (2004), Camera and Li (2008), Telyukova and Wright (2008), Sanches and Williamson (2010), Gu, Mattesini and Wright (2015), and Lotz and Zhang (2015).

${ }^{2}$ Carapella and Williamson (2015) consider similar credit-market frictions when exploring the beneficial roles of government debt. They show that the presence of government debt can improve welfare by relaxing credit constraints and by resolving an adverse selection problem. In their model, there is no
} 
we incorporate asymmetric information into a model of Lagos and Wright (2005) in which limited commitment is already embedded.

More specifically, in each period, a competitive market with search frictions (DM) and a frictionless centralized market $(\mathrm{CM})$ open sequentially. In the DM, half of the agents are buyers and half are sellers. There is an ex-ante heterogeneity across buyers in terms of CM productivities. ${ }^{3}$ Each buyer knows her productivity but the sellers cannot tell the difference. Buyers in the DM can borrow by issuing an IOU and sellers can make loans by taking IOUs in exchange for DM goods. Since there is no commitment or enforcement, a buyer issued an IOU in the previous DM can renege on her debt in the CM. Information about actions related to credit transactions is perfectly recorded and a buyer who reneges on her debt is permanently excluded from future DMs. It is noteworthy that as we will see later, this perfect credit-transaction information would not render money inessential under asymmetric information. That is, the wedge between unlimited monitoring of actions related to credit transactions and limited monitoring of actions related to borrowers' productivities is indeed in line with Wallace (2013): "To get money and credit, we need to have some monitoring - but not so much as to eliminate a role for money." 4

In the model, limited commitment yields an endogenous upper bound on the use of credit (a credit limit) for each borrower. However, the presence of asymmetric ingovernment-provided money and exchanges are carried out using credit only.

${ }^{3} \mathrm{Ex}$-ante heterogeneity is introduced in many microfounded search literature such as Boel and Camera (2009), Berentsen and Strub (2009), and Guerrieri, Shimer and Wright (2010).

${ }^{4}$ As shown in Kiyotaki and Wright $(1989,1993)$ and Kocherlakota (1998), among others, limited information and limited commitment are indispensable for the essentiality of money. In order to have the coexistence of money and credit, costly record-keeping that implies limited record-keeping is assumed in Camera and Li (2008), Gu, Mattesini and Wright (2015), Lotz and Zhang (2015), for instance. Another strand of literature exploring the coexistence imposes exogenous properties that render money or credit favorable. (See, for instance, He, Huang and Wright 2008, Kahn and Roberds 2008, and Sanches and Williamson 2010.) Besides the studies mentioned above, there are many other works dealing with the coexistence issue. For excellent surveys of related studies, see He, Huang and Wright (2008), Nosal and Rocheteau (2009), Gu, Mattesini and Wright (2015), Lagos, Rocheteau and Wright (2015), and Lotz and Zhang (2015). 
formation leads to an adverse selection problem such that the credit limit applied to a low-productivity borrower is applied to a high-productivity borrower also. A highproductivity borrower is then credit-constrained in the sense that the credit limit itself is insufficient to support her desired consumption. Therefore, a high-productivity borrower is willing to hold money to compensate for the deficiency of her credit limit. However, unlike a high-productivity borrower, a low-productivity borrower is not credit-constrained and is unwilling to hold money because the credit limit is sufficient to support her desired consumption.

All in all, the interplay among ex-ante heterogeneity, limited commitment, and asymmetric information eventually renders money and credit coessential. If there is no asymmetric information, limited commitment with perfect monitoring of credit transactions implies different self-enforcing credit limits for high-productivity and low-productivity buyers, respectively. In that case, money is inessential as in Kocherlakota (1998), Kocherlakota and Wallace (1998), and Wallace (2010, 2013), for instance. However, this result is overturned by the introduction of asymmetric information. Under asymmetric information in the credit market, our analysis shows that if the productivity difference between borrowers is large enough, the use of both money and credit improves social welfare. That is, money and credit are coessential at the aggregate transaction level in the sense that credit retains its role for improving the allocation of a low-productivity borrower while money retains its role for improving the allocation of a high-productivity borrower.

$\mathrm{Gu}$, Mattesini and Wright (2015) conjecture that the coessentiality of money and credit may arise when some of buyers do not demand money at all even when their consumption falls short of the first-best level. Our result suggests that this is indeed the case if there is an adverse selection problem in the credit market. Notice that our aggregate-level coessentiality with the buyer-side heterogeneity departs from related recent works such as Gomis-Porqueras and Sanches (2013) and Araujo and Hu (2014), which deliver individual- 
level coessentiality with the seller-side heterogeneity.

The paper proceeds as follows. Section 2 describes the background model. Section 3 presents benchmark results with exogenous credit limits. Section 4 endogenizes credit limits and explores the coessentiality of money and credit. Section 5 discusses the robustness of our result by considering an alternative information structure that allows sellers to access information about buyers' past actions perfectly. Section 6 summarizes the paper with a few concluding remarks.

\section{Model}

The background environment comes from Lagos and Wright (2005), and Rocheteau and Wright (2005). Time is discrete and there is a $[0,2]$ continuum of infinitely-lived agents. Each period is divided into two subperiods, in which trade occurs respectively in a Walrasian competitive market with search frictions (DM) and in a frictionless centralized market (CM). There is also an intrinsically useless, divisible, and durable object called money of which stock is exogenously given by $\mathbb{M}>0$. We here fix the stock of money over time to focus on ingredients of the coessentiality other than inflation. Each agent maximizes expected discounted utility with a discount factor $\beta \in(0,1)$.

In the DM, half of the agents can produce a DM good but do not want to consume it (hereinafter DM-sellers), while the remaining half want to consume one but cannot produce it (hereinafter DM-buyers). A DM good is perfectly divisible and perishable. An agent's identity as a DM-buyer or a DM-seller is permanent. DM-buyers get utility $u(q)$ from consuming $q$ units of DM goods where $u^{\prime \prime}(q)<0<u^{\prime}(q), u^{\prime}(0)=+\infty$, and $u^{\prime}(\infty)=u(0)=0$. DM-sellers incur disutility $q$ from producing $q$ units of DM goods.

As mentioned, the DM is a competitive market where search frictions are incorporated. Specifically, a competitive market opens at the beginning of the first subperiod and agents 
queue randomly to enter it. An agent entering the market observes the price $p$ announced by the Walrasian auctioneer and then chooses to either demand $q^{B}$ as a DM-buyer or supply $q^{S}$ as a DM-seller. The probability of joining the market for a DM-buyer is $\alpha \in(0,1)$ and that for a DM-seller is also $\alpha$ where $\alpha$ captures search frictions. After trading her money, a DM-buyer who has run out of money can borrow by issuing an IOU where an IOU is a promise to pay off a specified number of CM goods in the subsequent CM. A DM-seller can make loans by taking IOUs in exchange for DM goods. In doing that, DM-sellers cannot distinguish the CM productivities of IOU issuers. That is, as described below, there is an ex-ante heterogeneity across DM-buyers in terms of CM productivities. DM-sellers cannot observe buyers' productivities directly and cannot access information about buyers' past actions related to their productivities.

In the CM, all agents can consume and produce a CM good, which is perfectly divisible and perishable. The productivity of a $\theta \in(0,1)$ fraction of DM-buyers is relatively high (hereinafter $H$-type buyers), while that of a $(1-\theta)$ fraction of DM-buyers is relatively low (hereinafter $L$-type buyers). More specifically, an $H$-type buyer can produce a unit of CM good with $\left(1 / \varepsilon^{H}\right)$ units of labor which incur $\left(1 / \varepsilon^{H}\right)$ units of disutility, while an $L$-type buyer can produce a unit of CM good with $\left(1 / \varepsilon^{L}\right)$ units of labor which incur $\left(1 / \varepsilon^{L}\right)$ units of disutility where $\varepsilon^{H} \geq 1$ and $\varepsilon^{L} \leq 1$. A buyer's identity as an $H$-type buyer or an $L$-type buyer is permanent. (In Section 5, we also discuss the case in which a buyer's type follows a Markov process.) All DM-sellers are identical and can produce one unit of CM good with one unit of labor. Agents get utility $U(x)$ from consuming $x$ units of CM goods where $U^{\prime \prime}(x) \leq 0<U^{\prime}(x), U^{\prime}(0)=+\infty$, and $U^{\prime}(\infty)=0$. There is no commitment or enforcement and hence a DM-buyer issued an IOU in the previous DM can renege on her promise. In that case, the default is publicly announced and recorded: i.e., credit-transaction information is perfectly monitored and recorded. A buyer who defaults is then permanently excluded from future DMs. Notice that a defaulter can still 
produce CM goods for himself.

\section{Equilibrium}

We here focus on a stationary equilibrium in which the real balance of money in the CM is constant: i.e., for a given $\mathbb{M}, \phi_{t-1}=\phi_{t}=\phi$ where $\phi_{t}$ denotes the amount of CM good that one unit of money buys in the CM of period $t$. Hereinafter, we will drop the time subscript $t$ and index the next (previous) period by $+1(-1)$, if there is no risk of confusion.

As a stepping stone, we first define an equilibrium of a money-only economy in which credit is unavailable. Let $W(z)$ and $V(z)$ denote the expected value for an agent who enters the CM and the DM, respectively, with the real balance $z=\phi m$.

\subsection{Trades in the CM}

In the CM, all agents produce and consume CM goods, and demand money to be carried into the subsequent DM. Hence, the problem for an agent entering the CM with $z$ is

$$
\begin{gathered}
W^{j}(z)=\max _{\left(x_{M}^{j}, h^{j}, m_{+1}^{j}\right)} U\left(x_{M}^{j}\right)-\left(h^{j} / \varepsilon^{j}\right)+\beta V^{j}\left(\phi_{+1} m_{+1}^{j}\right) \\
\text { s.t. } x_{M}^{j}+\phi m_{+1}^{j}=h^{j}+z
\end{gathered}
$$

where $j \in\{H, L, S\}$ with $j=H$ denoting an $H$-type buyer $\left(\varepsilon^{H}=\varepsilon^{h}\right), j=L$ denoting an $L$-type buyer $\left(\varepsilon^{L}=\varepsilon^{l}\right)$, and $j=S$ denoting a DM-seller $\left(\varepsilon^{S}=1\right)$. We simply assume an interior solution for $x_{M}^{j}$ and $h^{j}$, respectively. Substituting $h^{j}$ from the constraint, we have

$$
W^{j}(z)=\frac{z}{\varepsilon^{j}}+\max _{\left(x_{M}^{j}, m_{+1}^{j}\right)}\left[U\left(x_{M}^{j}\right)-\frac{x_{M}^{j}}{\varepsilon^{j}}-\frac{\phi m_{+1}^{j}}{\varepsilon^{j}}+\beta V^{j}\left(\phi_{+1} m_{+1}^{j}\right)\right] .
$$


The first order conditions for $x_{M}^{j} \in \mathbb{R}_{++}, m_{+1}^{j} \in \mathbb{R}_{+}$, and the envelope condition are, respectively,

$$
\begin{gathered}
U^{\prime}\left(x_{M}^{j}\right)=1 / \varepsilon^{j} \\
\left(\phi / \varepsilon^{j}\right) \geq \beta \phi_{+1} V^{j^{\prime}}\left(\phi_{+1} m_{+1}^{j}\right),=\text { if } m_{+1}^{j}>0 \\
W^{j^{\prime}}=1 / \varepsilon^{j} .
\end{gathered}
$$

\subsection{Trades in the DM}

In the DM, taking $p \in \mathbb{R}_{++}$as given, a seller chooses $q_{M}^{S} \in \mathbb{R}_{++}$that solves

$$
\max _{q_{M}^{S}}\left[-q_{M}^{S}+W^{S}\left(\phi m+\phi p q_{M}^{S}\right)\right]
$$

which yields the optimality condition, $p \phi W^{S^{\prime}}=1$. The envelope condition in (3) together with $\varepsilon^{S}=1$ then implies that

$$
1=p \phi
$$

That is, DM-sellers produce up to the point at which the marginal cost of DM-good production is equal to its marginal revenue.

Similarly, taking $p \in \mathbb{R}_{++}$as given, a $j$-type buyer for $j \in\{H, L\}$ chooses $q_{M}^{j}$ that solves

$$
\max _{q_{M}^{j}}\left[u\left(q_{M}^{j}\right)+W^{j}\left(\phi m^{j}-\phi p q_{M}^{j}\right)\right]
$$

subject to $p q_{M}^{j} \leq m^{j}$. The first order condition for $q_{M}^{j} \in \mathbb{R}_{++}$is

$$
u^{\prime}\left(q_{M}^{j}\right)=\frac{1}{\varepsilon^{j}}\left(1+\varepsilon^{j} \lambda^{j}\right)
$$

where $\lambda^{j}$ is the Lagrangian multiplier on the budget constraint for $j \in\{H, L\}$, respectively. 
Now the value function for a $j$-type buyer is given by

$$
V^{j}\left(\phi m^{j}\right)=\max _{q_{M}^{j}} \alpha\left[u\left(q_{M}^{j}\right)+W^{j}\left(\phi m^{j}-\phi p q_{M}^{j}\right)\right]+(1-\alpha) W^{j}\left(\phi m^{j}\right)
$$

subject to $p q_{M}^{j} \leq m^{j}$ and the value function for a seller is given by

$$
V^{S}(\phi m)=\max _{q_{M}^{S}}\left[-q_{M}^{S}+W^{S}\left(\phi m+\phi p q_{M}^{S}\right)\right]
$$

The marginal value of holding money for a $j$-type buyer is then

$$
V^{j^{\prime}}\left(\phi m^{j}\right)= \begin{cases}1 / \varepsilon^{j} & \text { if } \lambda^{j}=0 \\ \alpha u^{\prime}\left(q_{M}^{j}\right)+(1-\alpha)\left(1 / \varepsilon^{j}\right) & \text { otherwise }\end{cases}
$$

which immediately suggests that a buyer is not willing to carry money into the DM so that $\lambda^{j}=0$ because in that case, the first order condition $(2)$ implies $1 / \beta=1$, which cannot happen for $\beta \in(0,1)$. Hence (2) eventually implies that the money demand of a $j$-type buyer in the CM is characterized by

$$
(r / \alpha)=\left[\varepsilon^{j} u^{\prime}\left(q_{M}^{j}\right)-1\right]
$$

where $r \equiv(1-\beta) / \beta$. Needless to say, the money demand of a seller in the CM is zero. We are now ready to define a monetary equilibrium of a money-only economy.

Definition 1 For a given $\mathbb{M}>0$, a stationary monetary equilibrium is a list $\left(q_{M}^{j}, x_{M}^{j}, \phi m_{+1}^{j}\right)$ for $j \in\{H, L, S\}$ such that: (i) $q_{M}^{j}$ for $j \in\{H, L\}$ solves $(6) ;($ ii $) q_{M}^{S}=\left[\theta q_{M}^{H}+(1-\theta) q_{M}^{L}\right]$; (iii) $x_{M}^{j}=x^{j *}=\arg \max \left[U\left(x^{j}\right)-\left(x^{j} / \varepsilon^{j}\right)\right]$ for $j \in\{H, L, S\}$ from $(1)$; (iv) $\phi m_{+1}^{j}=q_{M}^{j}>0$ for $j \in\{H, L\}$ with $m_{+1}^{S}=0$ and $\theta m_{+1}^{H}+(1-\theta) m_{+1}^{L}=\mathbb{M}$. 


\subsection{Exogenous Credit Limit and Coessentiality}

We now introduce an exogenous credit limit. Let $\bar{D}>0$ be an exogenous credit limit in terms of CM goods where $\bar{D}$ satisfies the voluntary repayment incentive constraints of buyers. Since credit is available in the DM, the state variables at the beginning of the CM are $(z, d) \in \mathbb{R}_{+} \times \mathbb{R}$ where $d$ is the real balances of debt used in the previous DM: i.e., it is a debt if $d$ is positive and a claim otherwise. $W^{j}(z, d)$ for $j \in\{H, L, S\}$ is then

$$
W^{j}(z, d)=\frac{z-d}{\varepsilon^{j}}+\max _{\left(x_{M C}^{j}, m_{+1}^{j}\right)}\left[U\left(x_{M C}^{j}\right)-\frac{x_{M C}^{j}}{\varepsilon^{j}}-\frac{\phi m_{+1}^{j}}{\varepsilon^{j}}+\beta V^{j}\left(\phi_{+1} m_{+1}^{j}\right)\right] .
$$

It is straightforward to show that the first order conditions for $x_{M C}^{j} \in \mathbb{R}_{++}$and $m_{+1}^{j} \in \mathbb{R}_{+}$ are the same as (1) and (2), respectively, and the envelope conditions are

$$
W_{1}^{j}=\left(1 / \varepsilon^{j}\right), \quad W_{2}^{j}=\left(-1 / \varepsilon^{j}\right)
$$

The state variable at the beginning of the DM is still $z^{j}=\phi m^{j}$ and $V^{j}\left(\phi m^{j}\right)$ for a $j$-type buyer is

$$
V^{j}\left(\phi m^{j}\right)=\max _{q_{M C}^{j}} \alpha\left\{u\left(q_{M C}^{j}\right)+W^{j}\left[\phi m^{j}-\left(\phi p q_{M C}^{j}-d_{b}^{j}\right), d_{b}^{j}\right]\right\}+(1-\alpha) W^{j}\left(\phi m^{j}, 0\right)
$$

subject to $\phi p q_{M C}^{j} \leq \phi m^{j}+d_{b}^{j}$ and $d_{b}^{j} \leq \bar{D}$. The value function for a seller is

$$
V^{S}(\phi m)=\max _{q_{M C}^{S}}\left[-q_{M C}^{S}+W^{S}\left(\phi m+\phi p q_{M C}^{S}+d_{s}, d_{s}\right)\right]
$$

Notice that even with $\bar{D}>0$, (6) still determines $q_{M C}^{j}$ for $j \in\{H, L\}$ if $m_{+1}^{j}>0$ and changes to the credit limit in the interval $\bar{D} \in\left(0, q_{M}^{L}\right)$ has no impact on $\left(q_{M C}^{H}, q_{M C}^{L}\right)$ because the real balance of money $\left(\phi m_{+1}^{j}\right)$ is endogenously adjusted to maintain a constant real balance. This is the neutrality result of credit in Gu, Mattesini and Wright (2015). The 
following lemma shows the condition under which a buyer in the CM demands money for the subsequent DM trades.

Lemma 1 For $j \in\{H, L\}, m_{+1}^{j}>0$ if $\bar{D}<q_{M}^{j}$ and $m_{+1}^{j}=0$ otherwise.

Lemma 1 just says that if the credit limit falls short of a buyer's desired expenditure in the subsequent DM, the marginal benefit from holding money is greater than its marginal cost and hence a buyer is willing to hold money.

Definition 2 For a given $(\mathbb{M}, \bar{D}) \in \mathbb{R}_{++}^{2}$, a stationary equilibrium is a list $\left(q_{M C}^{j}, x_{M C}^{j}, \phi m_{+1}^{j}\right)$ for $j \in\{H, L, S\}$ such that

(1) if $\bar{D}<q_{M}^{L}$, (i) $q_{M C}^{j}=q_{M}^{j}$ for $j \in\{H, L, S\}$, (ii) $x_{M C}^{j}=x_{M}^{j}=x^{j *}$ for $j \in\{H, L, S\}$, (iii) $\phi m_{+1}^{j}=q_{M C}^{j}-\bar{D}>0$ for $j \in\{H, L\}$ with $m_{+1}^{S}=0$ and $\theta m_{+1}^{H}+(1-\theta) m_{+1}^{L}=\mathbb{M}$;

(2) if $\bar{D} \in\left[q_{M}^{L}, q_{M}^{H}\right)$, (i) $q_{M C}^{H}=q_{M}^{H}$ and $q_{M C}^{L}=\min \left\{\bar{D}, q^{L *}\right\}$ where $q^{L *}=\arg \max \left\{u\left(q^{L}\right)-\right.$ $\left.\left(q^{L} / \varepsilon^{L}\right)\right\}$, (ii) $q_{M C}^{S}=\theta q_{M C}^{H}+(1-\theta) q_{M C}^{L}$, (iii) $x_{M C}^{j}=x_{M}^{j}=x^{j *}$ for $j \in\{H, L, S\}$, (iv) $\phi m_{+1}^{H}=q_{M C}^{H}-\bar{D}>0$ with $m_{+1}^{L}=m_{+1}^{S}=0$ and $\theta m_{+1}^{H}=\mathbb{M}$;

(3) if $\bar{D} \geq q_{M}^{H}$, (i) $q_{M C}^{j}=\min \left\{\bar{D}, q^{j *}\right\}$ for $j \in\{H, L\}$ where $q^{j *}=\arg \max \left\{u\left(q^{j}\right)-\right.$ $\left.\left(q^{j} / \varepsilon^{j}\right)\right\}$, (ii) $q_{M C}^{S}=\theta q_{M C}^{H}+(1-\theta) q_{M C}^{L}$, (iii) $x_{M C}^{j}=x_{M}^{j}=x^{j *}$ for $j \in\{H, L, S\}$, (iv) $\phi=0$ with $m_{+1}^{j}=0$ for $j \in\{H, L, S\}$.

Notice that in the equilibrium of (1) and (2) in Definition 2, both money and credit are circulated as means of exchange (a monetary equilibrium). However, in the equilibrium of (3) in Definition 2, only credit is circulated as a means of exchange because an exogenous credit limit is large enough to properly support $q_{M}^{H}$ and hence no one is willing to hold money (a nonmonetary equilibrium). This last type of an equilibrium immediately implies a credit-only allocation for a given $\bar{D}>0$. That is, for $j \in\{H, L\}, q_{C}^{j}=\bar{D}$ if $\bar{D}<q^{j *}$ 
because there is no money $(\mathbb{M}=0)$ and $q_{C}^{j}=q^{j *}$ if $\bar{D} \geq q^{j *}$ because the marginal benefit from increasing consumption beyond $q^{j *}$ is less than its marginal cost $\left(1 / \varepsilon^{j}\right)$.

Definition 3 For a given $\bar{D} \in \mathbb{R}_{++}$and $\mathbb{M}=0$, a stationary equilibrium is a list $\left(q_{C}^{j}, x_{C}^{j}\right)$ for $j \in\{H, L, S\}$ such that $(i) q_{C}^{j}=\min \left\{\bar{D}, q^{j *}\right\}$ for $j \in\{H, L\}$, (ii) $q_{C}^{S}=\theta q_{C}^{H}+$ $(1-\theta) q_{C}^{L}$, and $\left(\right.$ iii) $x_{C}^{j}=x_{M}^{j}=x^{j *}$ for $j \in\{H, L, S\}$.

Now in order to discuss coessentiality by which we mean that the use of both money and credit improves allocations from a social welfare perspective, we define social welfare $(\mathcal{W})$ as the steady-state sum of buyers' and sellers' utilities:

$$
\begin{aligned}
\mathcal{W}_{i}= & \alpha \theta\left[u\left(q_{i}^{H}\right)-q_{i}^{H}\right]+\alpha(1-\theta)\left[u\left(q_{i}^{L}\right)-q_{i}^{L}\right]+\theta\left[U\left(x^{H *}\right)-\left(x^{H *}+\alpha q_{i}^{H}\right) / \varepsilon^{H}\right]+ \\
& (1-\theta)\left[U\left(x^{L *}\right)-\left(x^{L *}+\alpha q_{i}^{L}\right) / \varepsilon^{L}\right]+\left[U\left(x^{S *}\right)-\left[x^{S *}-\alpha \theta q_{i}^{H}-\alpha(1-\theta) q_{i}^{L}\right]\right] \\
= & \alpha \theta\left[u\left(q_{i}^{H}\right)-\left(q_{i}^{H} / \varepsilon^{H}\right)\right]+\alpha(1-\theta)\left[u\left(q_{i}^{L}\right)-\left(q_{i}^{L} / \varepsilon^{L}\right)\right]+ \\
& \theta\left[U\left(x^{H *}\right)-\left(x^{H *} / \varepsilon^{H}\right)\right]+(1-\theta)\left[U\left(x^{L *}\right)-\left(x^{L *} / \varepsilon^{L}\right)\right]+\left[U\left(x^{S *}\right)-x^{S *}\right]
\end{aligned}
$$

where $i \in\{M, C, M C\}$ with $i=M$ denoting a money-only economy $(\mathbb{M}>0$ and $\bar{D}=0)$ of which allocation $\left(q_{M}^{H}, q_{M}^{L}, x_{M}^{H}, x_{M}^{L}, x_{M}^{S}\right)$ comes from Definition $1, i=C$ denoting a creditonly economy $(\mathbb{M}=0$ and $\bar{D}>0)$ of which allocation $\left(q_{C}^{H}, q_{C}^{L}, x_{C}^{H}, x_{C}^{L}, x_{C}^{S}\right)$ comes from Definition 3, and $i=M C$ denoting a money-credit economy $(\mathbb{M}>0$ and $\bar{D}>0)$ of which allocation $\left(q_{M C}^{H}, q_{M C}^{L}, x_{M C}^{H}, x_{M C}^{L}, x_{M C}^{S}\right)$ comes from Definition 2 .

Definition 4 Money and credit are coessential if $\mathcal{W}_{M C}>\mathcal{W}_{M}$ and $\mathcal{W}_{M C}>\mathcal{W}_{C}$

Now the following proposition suggests that for some credit limit $(\bar{D})$, the use of both money and credit can indeed improve social welfare.

Proposition 1 If an exogenous credit limit is given by $\bar{D} \in\left(q_{M}^{L}, q_{M}^{H}\right)$, money and credit are coessential. 
Proof. For a given $\bar{D} \in\left(q_{M}^{L}, q_{M}^{H}\right), \mathcal{W}_{M C}>\mathcal{W}_{M}$ and $\mathcal{W}_{M C}>\mathcal{W}_{C}$ are immediate consequences of the following: (i) $q_{M C}^{H}=q_{M}^{H}>q_{C}^{H}$ where the first equality is due to the neutrality result in Gu, Mattesini and Wright (2015) and the second inequality comes from $\bar{D}<q_{M}^{H}<q^{H *}$ which implies $q_{C}^{H}=\min \left\{\bar{D}, q^{H *}\right\}=\bar{D}$, (ii) $q_{M C}^{L}=q_{C}^{L}>q_{M}^{L}$ where the first equality comes from $q_{M C}^{L}=\min \left\{\bar{D}, q^{L *}\right\}=q_{C}^{L}$ from Definition 2 and Definition 3, respectively, and the second inequality is because if $q_{C}^{L}=\min \left\{\bar{D}, q^{L *}\right\}=\bar{D}, q_{M}^{L}<\bar{D}=q_{C}^{L}$ and if $q_{C}^{L}=\min \left\{\bar{D}, q^{L *}\right\}=q^{L *}, q_{M}^{L}<q^{L *}=q_{C}^{L}$, (iii) $\left[u\left(q^{j}\right)-\left(q^{j} / \varepsilon^{j}\right)\right]$ is strictly increasing for $q^{j} \in\left(0, q^{j *}\right)$, and (iv) $x_{M}^{j}=x_{C}^{j}=x_{M C}^{j}=x^{j *}$ for $j \in\{H, L, S\}$.

Intuitively, from the perspective of an $H$-type buyer, a money-only allocation $q_{M}^{H}$ cannot be attained with credit only and then $x_{M}^{H}=x_{C}^{H}=x^{H *}$ implies $\mathcal{W}_{M}^{H}=\alpha \theta\left[u\left(q_{M}^{H}\right)-\right.$ $\left.\left(q_{M}^{H} / \varepsilon^{H}\right)\right]+\theta\left[U\left(x^{H *}\right)-\left(x^{H *} / \varepsilon^{H}\right)\right]>\mathcal{W}_{C}^{H}=\alpha \theta\left[u\left(q_{C}^{H}\right)-\left(q_{C}^{H} / \varepsilon^{H}\right)\right]+\theta\left[U\left(x^{H *}\right)-\left(x^{H *} / \varepsilon^{H}\right)\right]$. In addition, even if both money and credit are available with credit limit $\bar{D}$ such that $\bar{D}<q_{M}^{H}$, the DM-consumption for an $H$-type buyer still stays at $q_{M}^{H}$ and hence there is no role for credit in improving the allocation of an $H$-type buyer $\left(\mathcal{W}_{M}^{H}=\mathcal{W}_{M C}^{H}\right)$. In the end, money is essential but credit is inessential for an $H$-type buyer. Meanwhile, from the perspective of an $L$-type buyer, a credit-only allocation $q_{C}^{L}$ cannot be attained with money only and then $x_{M}^{L}=x_{C}^{L}=x^{L *}$ implies $\mathcal{W}_{C}^{L}=\alpha(1-\theta)\left[u\left(q_{C}^{L}\right)-\left(q_{C}^{L} / \varepsilon^{L}\right)\right]+(1-\theta)\left[U\left(x^{L *}\right)-\left(x^{L *} / \varepsilon^{L}\right)\right]>$ $\mathcal{W}_{M}^{L}=\alpha(1-\theta)\left[u\left(q_{M}^{L}\right)-\left(q_{M}^{L} / \varepsilon^{L}\right)\right]+(1-\theta)\left[U\left(x^{L *}\right)-\left(x^{L *} / \varepsilon^{L}\right)\right]$. In addition, even if both money and credit are available, the DM-consumption for an $L$-type buyer still stays at $q_{C}^{L}=\min \left\{\bar{D}, q^{L *}\right\}$ and hence there is no role for money in improving the allocation of an $L$-type buyer $\left(\mathcal{W}_{C}^{L}=\mathcal{W}_{M C}^{L}\right)$. Consequently, credit is essential but money is inessential for an $L$-type buyer. Noting that $\mathcal{W}_{M}^{S}=\left[U\left(x^{S *}\right)-x^{S *}\right]=\mathcal{W}_{C}^{S}=\mathcal{W}_{M C}^{S}$, all these together finally imply that money and credit are coessential at the aggregate transaction level if $\bar{D} \in\left(q_{M}^{L}, q_{M}^{H}\right)$ : i.e., $\mathcal{W}_{M C}=\mathcal{W}_{M C}^{H}+\mathcal{W}_{M C}^{L}+\mathcal{W}_{M C}^{S}>\mathcal{W}_{M}=\mathcal{W}_{M}^{H}+\mathcal{W}_{M}^{L}+\mathcal{W}_{M}^{S}$ and $\mathcal{W}_{M C}=\mathcal{W}_{M C}^{H}+\mathcal{W}_{M C}^{L}+\mathcal{W}_{M C}^{S}>\mathcal{W}_{C}=\mathcal{W}_{C}^{H}+\mathcal{W}_{C}^{L}+\mathcal{W}_{C}^{S}$ 


\section{Endogenous Credit Limit}

We now endogenize the credit limit based on the approach in $\mathrm{Gu}$, Mattesini and Wright (2015). We start with an arbitrary credit limit $D^{j}$ for a $j$-type buyer. Notice that the value for a non-default buyer can be expressed as

$$
W^{j}(z, d)=\frac{z-d}{\varepsilon^{j}}+U\left(x^{j *}\right)-\frac{x^{j *}}{\varepsilon^{j}}-\frac{\phi m_{+1}^{j}}{\varepsilon^{j}}+\beta V^{j}\left(\phi_{+1} m_{+1}^{j}\right)
$$

If a buyer defaults, she can enjoy more leisure but cannot enter future DMs. Hence, the value for a defaulter is

$$
\bar{W}^{j}(z, d)=\frac{z}{\varepsilon^{j}}+U\left(x^{j *}\right)-\frac{x^{j *}}{\varepsilon^{j}}+\beta \bar{V}^{j}(0)
$$

where $\bar{V}^{j}(0)$ is her value at the beginning of the subsequent DM. We let $\mathcal{L}^{j}\left(D^{j}\right)$ denote the loss from reneging on debt for a $j$-type buyer for a given $D^{j}, \mathcal{L}^{j}\left(D^{j}\right) \equiv W^{j}(\cdot)-\bar{W}^{j}(\cdot)$. Then $\mathcal{L}^{j}\left(D^{j}\right)$ for $j \in\{H, L\}$ is given by

$$
\mathcal{L}^{j}\left(D^{j}\right)= \begin{cases}\left(\frac{\alpha}{r}\right) u\left(q_{M}^{j}\right)-\left(1+\frac{\alpha}{r}\right) \frac{q_{M}^{j}}{\varepsilon^{j}} & \text { if } D^{j}<q_{M}^{j} \\ \left(\frac{\alpha}{r}\right) u\left(D^{j}\right)-\left(1+\frac{\alpha}{r}\right) \frac{D^{j}}{\varepsilon^{j}} & \text { if } q_{M}^{j} \leq D^{j}<q^{j *} \\ \left(\frac{\alpha}{r}\right)\left[u\left(q^{j *}\right)-\frac{q^{j *}}{\varepsilon^{j}}\right]-\frac{D^{j}}{\varepsilon^{j}} & \text { if } q^{j *} \leq D^{j}\end{cases}
$$

where we use $q_{M}^{j}=D^{j}+\phi m_{+1}^{j}$ for $D^{j}<q_{M}^{j}$ from Definition 2 .

Now, for the discussion of an endogenous credit limit, it is convenient to define $\Phi^{j}\left(D^{j}\right)=\mathcal{L}^{j}\left(D^{j}\right)+\left(D^{j} / \varepsilon^{j}\right)$ as in $\mathrm{Gu}$, Mattesini and Wright (2015). That is, $\Phi^{j}\left(D^{j}\right)$ can be expressed as 


$$
\Phi^{j}\left(D^{j}\right)= \begin{cases}\left(\frac{\alpha}{r}\right) u\left(q_{M}^{j}\right)-\left(1+\frac{\alpha}{r}\right) \frac{q_{M}^{j}}{\varepsilon^{j}}+\frac{D^{j}}{\varepsilon^{j}} & \text { if } D^{j}<q_{M}^{j} \\ \left(\frac{\alpha}{r}\right)\left[u\left(D^{j}\right)-\frac{D^{j}}{\varepsilon^{j}}\right] & \text { if } q_{M}^{j} \leq D^{j}<q^{j *} \\ \left(\frac{\alpha}{r}\right)\left[u\left(q^{j *}\right)-\frac{q^{j *}}{\varepsilon^{j}}\right] & \text { if } q^{j *} \leq D^{j}\end{cases}
$$

and then an endogenous credit limit for a $j$-type buyer $\left(\hat{D}^{j}\right)$ is simply a nonnegative fixed point $\hat{D}^{j}=\varepsilon^{j} \Phi^{j}\left(\hat{D}^{j}\right)$. The following proposition shows that the credit limit for each type $j \in\{H, L\}$ is always well defined.

Proposition 2 For each $j \in\{H, L\}$, there is a unique $\hat{D}^{j}>q_{M}^{j}$ such that $\hat{D}^{j}=\varepsilon^{j} \Phi^{j}\left(\hat{D}^{j}\right)$.

Proof. Notice first that $\varepsilon^{j} \Phi^{j}\left(D^{j}\right)$ for $j \in\{H, L\}$ is continuous and weakly increasing: that is, (i) for $D^{j}<q_{M}^{j}, \varepsilon^{j} \Phi^{j}\left(D^{j}\right)$ increases with a slope of 1 , (ii) for $q_{M}^{j} \leq D^{j}<$ $q^{j *}$, the slope of $\varepsilon^{j} \Phi^{j}\left(D^{j}\right)$ is $(\alpha / r)\left[\varepsilon^{j} u^{\prime}\left(D^{j}\right)-1\right]$ which is strictly positive because $D^{j}<$ $q^{j *}$ and decreases as $D^{j}$ increases because $u^{\prime \prime}<0$, and (iii) for $q^{j *} \leq D^{j}, \varepsilon^{j} \Phi^{j}\left(D^{j}\right)$ is constant. Notice also that from $(10), \Phi^{j}(0)=(\alpha / r)\left\{u\left(q_{M}^{j}\right)-\left(1 / \varepsilon^{j}\right)[(\alpha+r) / \alpha] q_{M}^{j}\right\}$. Now $u^{\prime}\left(q_{M}^{j}\right)=\left(1 / \varepsilon^{j}\right)[(\alpha+r) / \alpha]$ from (6) implies that at $q_{M}^{j}$, the slope of $u\left(q_{M}^{j}\right)$ and that of $\left(1 / \varepsilon^{j}\right)[(\alpha+r) / \alpha] q_{M}^{j}$ are the same. Then $\Phi^{j}(0)>0$ is an immediate consequence of $u^{\prime}(0)=\infty$ and $u^{\prime \prime}<0$. All these together finally imply an existence of a unique fixed point $\hat{D}^{j}=\varepsilon^{j} \Phi^{j}\left(\hat{D}^{j}\right)$ such that $\hat{D}^{j}>q_{M}^{j}$ for each $j \in\{H, L\}$. (See Figure 1 for an illustration.)

Now, the following proposition shows that if there is no asymmetric information on the productivities of borrowers and monitoring on credit transactions is perfect, money is inessential. This result is well-established in the existing literature such as, for instance, Kocherlakota (1998), Kocherlakota and Wallace (1998), and Wallace (2010).

Proposition 3 If there is no asymmetric information in the credit market, money is inessential. 


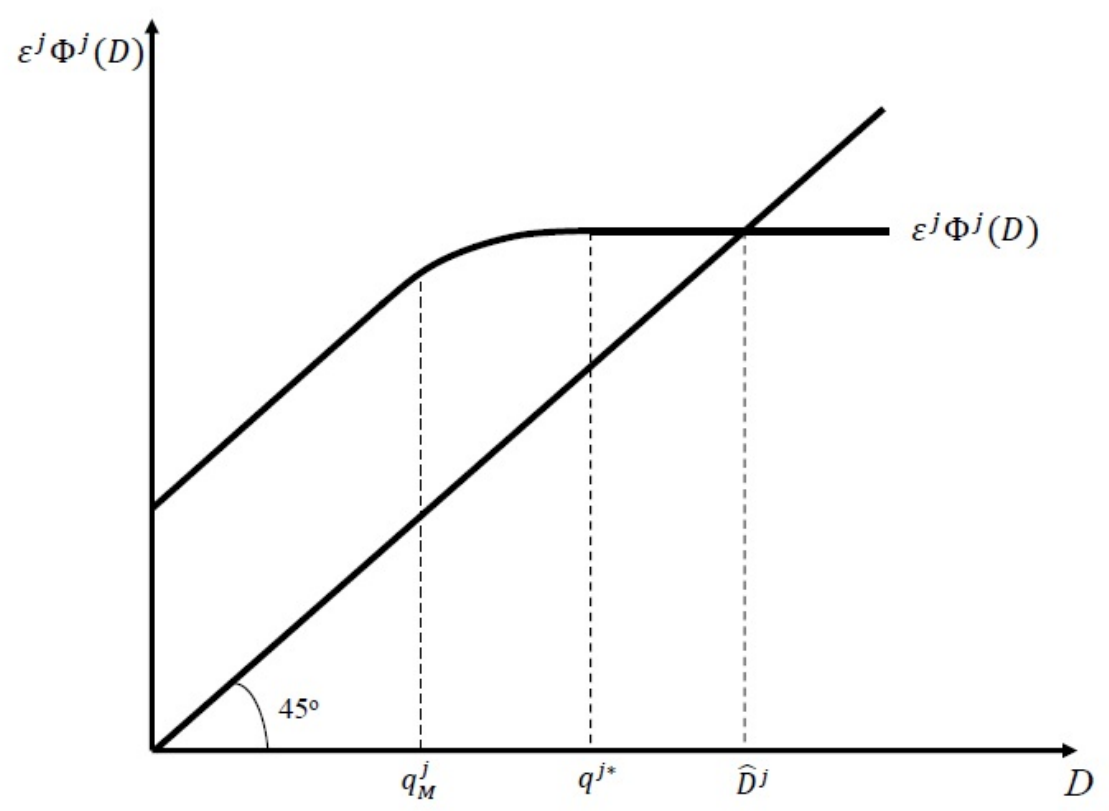

Figure 1: An illustration of a fixed point

Proof. From Proposition 2, a unique fixed point $\hat{D}^{j}$ for each $j \in\{H, L\}$ is greater than $q_{M}^{j}$. Then credit-only allocation $q_{C}^{j}=\min \left\{\hat{D}^{j}, q^{j *}\right\}$ for each $j \in\{H, L\}$ cannot be attained with money only and hence $\mathcal{W}_{C}>\mathcal{W}_{M}$. In addition, even if both money and credit are available, the DM-consumption for a $j$-type buyer still stays at $q_{C}^{j}=\min \left\{\hat{D}^{j}, q^{j *}\right\}$ because no one is willing to hold money and hence there is no role for money in improving allocations $\left(\mathcal{W}_{C}=\mathcal{W}_{M C}\right)$. These together imply that money is inessential if there is no asymmetric information.

We now examine what happens to the result in Proposition 3 if there is asymmetric information in the credit market. That is, there is an ex-ante heterogeneity on the productivities of buyers (borrowers). Buyers know their own productivities but sellers (lenders) cannot identify the productivities of buyers. Sellers then care only about an $L$-type buyer's credit limit: i.e., sellers who are unable to identify $H$-type buyers from $L$-type buyers are unwilling to extend credit beyond $\hat{D}^{L}$. 
Lemma 2 If there is asymmetric information in the credit market, sellers will make loans up to $\hat{D}^{L}$ such that $\hat{D}^{L}=\varepsilon^{L} \Phi^{L}\left(\hat{D}^{L}\right)$.

Proof. Since a seller cannot distinguish an $H$-type buyer from an $L$-type buyer, she is willing to extend the loan $D$ if

$$
D \leq\left[\theta \mathbb{I}_{H}+(1-\theta) \mathbb{I}_{L}\right] D
$$

where $\mathbb{I}_{j}=1$ for $j \in\{H, L\}$ if a $j$-type borrower honors her loans and $\mathbb{I}_{j}=0$ otherwise. The left-hand side of (11) is the cost of making loan $D$ and the right-hand side is its expected revenue. Since $\left(\mathbb{I}_{H}, \mathbb{I}_{L}\right)=(0,0)$ for $D>\hat{D}^{H}$ and $\left(\mathbb{I}_{H}, \mathbb{I}_{L}\right)=(1,0)$ for $D \in$ $\left(\hat{D}^{L}, \hat{D}^{H}\right]$ where $\hat{D}^{j}$ for $j \in\{H, L\}$ comes from Proposition 2, an upper bound of $D$ satisfying (11) is given by $\hat{D}^{L}$.

This is an adverse selection problem due to asymmetric information. Since $H$-type buyers are then also subject to $\hat{D}^{L}$, it is possible that $\hat{D}^{L} \in\left(q_{M}^{L}, q_{M}^{H}\right)$. The following proposition shows that this indeed happens if $\varepsilon^{H}$ is large enough relative to $\varepsilon^{L}$.

Proposition 4 Suppose there is asymmetric information in the credit market. If $\left(\varepsilon^{H} / \varepsilon^{L}\right)$ is large enough, L-type buyers use credit only while $H$-type buyers use both money and credit in equilibrium.

Proof. We first fix $\varepsilon^{L}$. Notice that $\hat{D}^{L}>q_{M}^{L}$ from Proposition 2. Suppose then $q_{M}^{L}<\hat{D}^{L}<q^{L *}$. If $\varepsilon^{H}=\varepsilon^{L}, q_{M}^{H}=q_{M}^{L}$ and hence $q_{M}^{H}<\hat{D}^{L}$. As $\varepsilon^{H}$ increases from $\varepsilon^{L}, q_{M}^{H}$ also increases from (6) but $\hat{D}^{L}$ remains constant because $\hat{D}^{L}$ is irrelevant to $\varepsilon^{H}$. Hence $\bar{\varepsilon}_{1}^{H}$ that satisfies $q_{M}^{H}=\hat{D}^{L}$ is well defined. Now if $\varepsilon^{H}>\bar{\varepsilon}_{1}^{H}$, an $L$-type buyer uses only credit up to $\hat{D}^{L}$ because $q_{M}^{L}<\hat{D}^{L}<q^{L *}$ and a $H$-type buyer uses both money and credit because $\hat{D}^{L}<q_{M}^{H}$. Suppose now $q_{M}^{L}<q^{L *} \leq \hat{D}^{L}$. If $\varepsilon^{H}=\varepsilon^{L}, q_{M}^{H}=q_{M}^{L}$ and hence $q_{M}^{H}<\hat{D}^{L}$. Notice again that $q_{M}^{H}$ increases with $\varepsilon^{H}$ but $\hat{D}^{L}$ remains constant. Therefore, 
there is $\bar{\varepsilon}_{2}^{H}$ that satisfies $q_{M}^{H}=\hat{D}^{L}$ and if $\varepsilon^{H}>\bar{\varepsilon}_{2}^{H}$, an $L$-type buyer uses only credit up to $q^{L *}$ because $\hat{D}^{L} \geq q^{L *}$ and an $H$-type buyer uses both money and credit because $\hat{D}^{L}<q_{M}^{H}$

Intuitively, since $q_{M}^{H}$ increases with $\varepsilon^{H}$ from (6) and $\hat{D}^{L}>q_{M}^{L}$ from Proposition 2, it can be the case that $q_{M}^{L}<\hat{D}^{L}<q_{M}^{H}$ if the productivity of an $H$-type buyer is large enough compared to that of an $L$-type buyer. Proposition 4 together with Proposition 1 now implies the coessentiality of money and credit at the aggregate transaction level as in the previous section with an exogenous credit limit.

Corollary 1 If the assumptions in Proposition 4 hold, money and credit are coessential.

Proof. Under the assumptions in Proposition 4, the credit limit for a money-credit economy satisfies $q_{M}^{L}<\hat{D}^{L}<q_{M}^{H}$. Meanwhile, the credit limit in a credit-only economy can be defined as a nonnegative fixed point $\hat{D}_{C}^{L}=\varepsilon^{L} \Phi_{C}^{L}\left(\hat{D}_{C}^{L}\right)$ where $\Phi_{C}^{L}\left(D_{C}^{L}\right)$ is given by

$$
\Phi_{C}^{L}\left(D_{C}^{L}\right)= \begin{cases}\left(\frac{\alpha}{r}\right)\left[u\left(D_{C}^{L}\right)-\frac{D_{C}^{L}}{\varepsilon^{L}}\right] & \text { if } D_{C}^{L}<q^{L *} \\ \left(\frac{\alpha}{r}\right)\left[u\left(q^{L *}\right)-\frac{q^{L *}}{\varepsilon^{L}}\right] & \text { if } q^{L *} \leq D_{C}^{L} .\end{cases}
$$

Then, there are two fixed points $\hat{D}_{C}^{L}$ : one is zero $\left(\hat{D}_{C}^{L}=0\right)$ because $\Phi_{C}^{L}(0)=0$ and the other is strictly positive $\left(\hat{D}_{C}^{L}>0\right)$. For the latter, $\hat{D}_{C}^{L}=\hat{D}^{L}$ because $\varepsilon^{L} \Phi^{L}(D)=\varepsilon^{L} \Phi_{C}^{L}(D)$ for $D \geq q_{M}^{L}$ and there is no fixed point $\hat{D}^{L}$ in the interval $\left(0, q_{M}^{L}\right)$ from Proposition 2. Exactly the same arguments in Proposition 1 then imply that a money-credit allocation $\left(q_{M C}^{H}, q_{M C}^{L}, x_{M C}^{H *}, x_{M C}^{L *}, x_{M C}^{S *}\right)$ dominates a money-only allocation $\left(q_{M}^{H}, q_{M}^{L}, x_{M}^{H *}, x_{M}^{L *}, x_{M}^{S *}\right)$ and also a credit-only allocation $\left(q_{C}^{H}, q_{C}^{L}, x_{C}^{H *}, x_{C}^{L *}, x_{C}^{S *}\right)$ in terms of social welfare: i.e., $\mathcal{W}_{M C}>$ $\mathcal{W}_{M}$ and $\mathcal{W}_{M C}>\mathcal{W}_{C}$. Obviously, this result remains intact even in the case of $\hat{D}_{C}^{L}=0$ because $\mathcal{W}_{M}$ is irrelevant to a credit limit and $q_{C}^{j}=0$ for $j \in\{H, L\}$ if $\hat{D}_{C}^{L}=0$. 
In sum, the main friction leading to our coessentiality result is asymmetric information which triggers an adverse selection problem such that the credit limit for a lowproductivity borrower is also imposed on a high-productivity borrower. Thus, a highproductivity borrower can be credit-constrained in the sense that the credit limit itself is insufficient to support her desired level of consumption, while a low-productivity borrower is not. Then, a high-productivity borrower is willing to hold money to compensate for the deficiency of her credit limit, whereas a low-productivity borrower uses credit only and does not demand money at all. An example of a money-credit equilibrium following Proposition 4 is given below.

Example Suppose $u(q)=2 \sqrt{q}, \alpha=1 / 2, r=(1-\beta) / \beta=0.04$, and $\varepsilon^{L}=1$. For these parameters, we are going to construct an equilibrium corresponding to Proposition 4 where an L-type buyer uses credit up to $q^{L *}$ and an $H$-type buyer uses both money and credit to consume $q_{M}^{H}$ which is greater than $\hat{D}^{L}$. First notice that by the construction, $q^{L *}=1$ in this equilibrium and $\hat{D}^{L}=(\alpha / r)\left[u\left(q^{L *}\right)-q^{L *}\right]=(25 / 2)(2-1)=12.5$. We then need to check whether there exists $\bar{\varepsilon}_{2}^{H}$ such that for $\varepsilon^{H}>\bar{\varepsilon}_{2}^{H}, q_{M}^{H}>\hat{D}^{L}>q^{L *}=1$. This inequality holds if $\varepsilon^{H}>3.82=\bar{\varepsilon}_{2}^{H}$ because $q_{M}^{H}=\left\{\varepsilon^{H} /[1+(r / \alpha)]\right\}^{2}$ from (6).

\section{Perfect Information on Buyers' Past Actions}

We now discuss the generality of our result by taking into account an alternative information structure that allows sellers to access information about buyers' past actions perfectly. In the baseline model, we assume that information about actions related to credit transactions is perfectly monitored and recorded, but information about actions related to buyers' productivities is not recorded at all. This imperfect information on buyers' past actions is assumed to incorporate asymmetric information into an environment where a buyer's type is permanent. If a buyer's type is not permanent, this assumption of imperfect 
information would not be necessary.

In order to see this point, consider a simple variation of our baseline model in which a buyer's type follows a Markov process such that a $j$-type buyer in the current CM remains a $j$-type buyer in the next $\mathrm{CM}$ with probability $\pi$ but becomes another type buyer with probability $1-\pi$ : i.e., unlike the baseline model, a buyer's type $j \in\{H, L\}$ is not permanent but varies over time. Each buyer recognizes her type at the beginning of the DM. For any $\pi \in(0,1), \theta_{t=0}=1 / 2$ then yields a time-invariant distribution such that at the beginning of DM, $\left(\theta_{t}, 1-\theta_{t}\right)=(1 / 2,1 / 2)$ for all $t$. Sellers can access information about buyers' past actions perfectly including money holdings.

In this environment, perfect information on buyers' past actions can reduce uncertainty but cannot completely resolve the adverse selection problem. That is, a buyer's money demand in the previous CM gives information related to whether she was an $H$-type or an $L$-type buyer. If she was an $H$-type, she is an $H$-type in the current DM with probability $\pi$ and an $L$-type with probability $(1-\pi)$. A seller is then willing to extend loan $D$ to her if $D \leq\left[\pi \mathbb{I}_{H}+(1-\pi) \mathbb{I}_{L}\right] D$ where $\mathbb{I}_{j}=1$ for $j \in\{H, L\}$ if a $j$-type buyer honors her loans and $\mathbb{I}_{j}=0$ otherwise. The left-hand side of the condition above is the cost of making loan $D$ to the buyer and the right-hand side is its expected revenue. Let $\hat{D}^{j}$ for $j \in\{H, L\}$ be the endogenous credit limit for a $j$-type buyer as defined in Section 4 . Then since $\left(\mathbb{I}_{H}, \mathbb{I}_{L}\right)=(0,0)$ for $D>\hat{D}^{H}$ and $\left(\mathbb{I}_{H}, \mathbb{I}_{L}\right)=(1,0)$ for $D \in\left(\hat{D}^{L}, \hat{D}^{H}\right]$, any $D>\hat{D}^{L}$ cannot meet the condition above. Exactly the same argument can be applied to a buyer who was an $L$-type in the previous CM.

Now the following proposition shows that if $\pi$ is large enough, the result in Proposition 4 still holds even if sellers can observe buyers' past actions perfectly.

Proposition 5 Suppose sellers can access information about buyers' past actions perfectly. If the assumptions in Proposition 4 hold and $\pi$ is sufficiently close to 1, L-type 
buyers use credit only while $H$-type buyers use both money and credit in equilibrium.

Proof. See Appendix.

This result with Corollary 1 implies that imperfect information on buyers' past actions is not crucial for the coessentiality result. More to the point is the adverse selection problem due to asymmetric information and our coessentiality result is its immediate con-

sequence. Put another way, the coessentiality result will remain intact if the monitoring and recording-keeping of individual actions do not significantly help resolve the asymmetric information problem. This alludes to the idea that in a heterogeneous-agent model, incomplete information on agents' types might generate an essentiality of money without relying on imperfect information on individual actions.

\section{Concluding Remarks}

Most of the existing literature relevant to our paper mainly focused on the coexistence rather than the coessentiality of money and credit. But Gu, Mattesini and Wright (2015) have shown that the coexistence does not necessarily imply the coessentiality. They then conjecture that coessentiality occurs if some of buyers do not demand money at all even if their consumption falls short of the first-best level. We show that this is indeed the case if there is asymmetric information in the credit market.

Several works have explored the coessentiality itself, including Berentsen, Camera and Waller (2007), Gomis-Porqueras and Sanches (2013), and Araujo and $\mathrm{Hu}$ (2014). Our paper adds to this literature asymmetric information as one of the frictions that renders money and credit coessential. In doing that, rather than use an innovative model, we take an established off-the-shelf model and incorporate credit market frictions to see their importance in explaining the coessentiality of money and credit. Through this natural way, we find that asymmetric information matters a great deal for coessentiality. This is 
why we believe that private information in the credit market is indeed one of the crucial factors for understanding the coessentiality of money and credit.

\section{Appendix: Proof of Proposition 5}

We here show Proposition 5 by way of a constructive proof. We start with the equilibrium

characterized in Proposition 4. Choose first an arbitrarily $D^{L}$ such that $q^{L *}<D^{L}<q_{M}^{H}$. Then, for a given $\pi$, the value for a non-default $L$-type buyer is

$$
W^{L}\left(z, D^{L} ; \pi\right)=U\left(x^{L *}\right)-\left(1 / \varepsilon^{L}\right)\left(D^{L}+x^{L *}-z\right)+\beta\left[\pi V^{L L}(0)+(1-\pi) V^{L H}(0)\right] .
$$

Here $V^{j k}$ is the value at the beginning of the next DM with $j(k)$ denoting the currentperiod (the next-period) type and

$$
\begin{aligned}
V^{L L}(0)= & \alpha u\left(q^{L *}\right)+U\left(x^{L *}\right)-\left(\alpha q^{L *}+x^{L *}\right) / \varepsilon^{L}+\beta\left[\pi V^{L L}(0)+(1-\pi) V^{L H}(0)\right] \\
V^{L H}(0)= & \alpha u\left(D^{L}\right)+U\left(x^{H *}\right)-\left(\alpha D^{L}+x^{H *}+\phi m_{+1}^{H}\right) / \varepsilon^{H} \\
& +\beta\left[\pi V^{H H}\left(\phi m_{+1}^{H}\right)+(1-\pi) V^{H L}\left(\phi m_{+1}^{H}\right)\right] \\
V^{H L}\left(\phi m^{H}\right)= & \alpha u\left(q^{L *}\right)+U\left(x^{L *}\right)-\left(\alpha q^{L *}+x^{L *}-\phi m^{H}\right) / \varepsilon^{L} \\
& +\beta\left[\pi V^{L L}(0)+(1-\pi) V^{L H}(0)\right] \\
V^{H H}\left(\phi m^{H}\right) & \alpha u\left[q_{M}^{H}(\pi)\right]+U\left(x^{H *}\right)-\left[\alpha\left(D^{L}+\phi m_{+1}^{H}\right)+x^{H *}\right] / \varepsilon^{H} \\
& +\beta\left[\pi V^{H H}\left(\phi m_{+1}^{H}\right)+(1-\pi) V^{H L}\left(\phi m_{+1}^{H}\right)\right]
\end{aligned}
$$

where for a given $D^{L}$, as in $(6), \phi m_{+1}^{H}$ is characterized by $(1 / \beta)=\pi\left[\alpha \varepsilon^{H} u^{\prime}\left(\phi m_{+1}^{H}+D^{L}\right)+\right.$ $(1-\alpha)]+(1-\pi)\left(\varepsilon^{H} / \varepsilon^{L}\right)$. Notice that currently $L$-type buyers do not hold money because the marginal benefit of holding money is less than its marginal cost: 


$$
\begin{aligned}
& \beta \phi\left\{\frac{\pi}{\varepsilon^{L}}+(1-\pi)\left[\alpha u^{\prime}\left(D^{L}\right)+\frac{1-\alpha}{\varepsilon^{H}}\right]\right\}<\beta \phi\left\{\frac{\pi}{\varepsilon^{L}}+(1-\pi)\left[\alpha u^{\prime}\left(q^{L *}\right)+\frac{1-\alpha}{\varepsilon^{H}}\right]\right\} \\
& =\left(\frac{\beta \phi}{\varepsilon^{L}}\right)\left[\pi+(1-\pi) \alpha+(1-\pi)(1-\alpha)\left(\frac{\varepsilon^{L}}{\varepsilon^{H}}\right)\right]<\frac{\beta \phi}{\varepsilon^{L}}<\frac{\phi}{\varepsilon^{L}} .
\end{aligned}
$$

Now the value for an $L$-type defaulter for a given $\pi$ can be expressed as

$$
\bar{W}^{L}\left(z, D^{L} ; \pi\right)=U\left(x^{L *}\right)-\left[\left(x^{L *}-z\right) / \varepsilon^{L}\right]+\beta\left[\pi \bar{V}^{L}(0)+(1-\pi) \bar{V}^{H}(0)\right]
$$

where $\bar{V}^{L}(0)$ and $\bar{V}^{H}(0)$ are respectively given by

$$
\begin{aligned}
& \bar{V}^{L}(0)=U\left(x^{L *}\right)-\left(x^{L *} / \varepsilon^{L}\right)+\beta\left[\pi \bar{V}^{L}(0)+(1-\pi) \bar{V}^{H}(0)\right] \\
& \bar{V}^{H}(0)=U\left(x^{H *}\right)-\left(x^{H *} / \varepsilon^{H}\right)+\beta\left[\pi \bar{V}^{H}(0)+(1-\pi) \bar{V}^{L}(0)\right] .
\end{aligned}
$$

By solving recursively, we then have

$$
\begin{aligned}
V^{L L}(0) & =\left(\Delta \frac{\beta^{3} \pi(1-\pi)^{2}}{(1-\beta \pi)^{3}}+\frac{1}{1-\beta \pi}\right)\left[\alpha u\left(q^{L *}\right)+U\left(x^{L *}\right)-\left(\alpha q^{L *}+x^{L *}\right) / \varepsilon^{L}\right] \\
& +\left(\Delta \frac{\beta^{3}(1-\pi)^{3}}{(1-\beta \pi)^{3}}+\frac{\beta(1-\pi)}{1-\beta \pi}\right)\left[\alpha u\left(D^{L}\right)+U\left(x^{H *}\right)-\left[x^{H *}+q_{M}^{H}(\pi)-(1-\alpha) D^{L}\right] / \varepsilon^{H}\right] \\
& +\left(\Delta \frac{\beta^{2}(1-\pi)^{2}}{(1-\beta \pi)^{2}}\right)\left[u\left(q^{L *}\right)+U\left(x^{L *}\right)-\left[\alpha q^{L *}+x^{L *}-\left(q_{M}^{H}(\pi)-D^{L}\right)\right] / \varepsilon^{L}\right] \\
& +\left(\Delta \frac{\beta^{4} \pi(1-\pi)^{3}}{(1-\beta \pi)^{4}}+\frac{\beta^{2} \pi(1-\pi)}{(1-\beta \pi)^{2}}\right)\left[\alpha u\left[q_{M}^{H}(\pi)\right]+U\left(x^{H *}\right)-\left[x^{H *}+\alpha q_{M}^{H}(\pi)\right] / \varepsilon^{H}\right] \\
V^{L H}(0) & =\Delta \frac{\beta^{2} \pi(1-\pi)}{(1-\beta \pi)^{2}}\left[\alpha u\left(q^{L *}\right)+U\left(x^{L *}\right)-\left(\alpha q^{L *}+x^{L *}\right) / \varepsilon^{L}\right] \\
& +\left(\Delta \frac{\beta^{2}(1-\pi)^{2}}{(1-\beta \pi)^{2}}+1\right)\left[\alpha u\left(D^{L}\right)+U\left(x^{H *}\right)-\left[x^{H *}+q_{M}^{H}(\pi)-(1-\alpha) D^{L}\right] / \varepsilon^{H}\right] \\
& +\left(\Delta \frac{\beta(1-\pi)}{(1-\beta \pi)}\right)\left[u\left(q^{L *}\right)+U\left(x^{L *}\right)-\left[\alpha q^{L *}+x^{L *}-\left(q_{M}^{H}(\pi)-D^{L}\right)\right] / \varepsilon^{L}\right] \\
& +\left(\Delta \frac{\beta^{3} \pi(1-\pi)^{2}}{(1-\beta \pi)^{3}}+\frac{\beta \pi}{(1-\beta \pi)}\right)\left[\alpha u\left[q_{M}^{H}(\pi)\right]+U\left(x^{H *}\right)-\left[x^{H *}+\alpha q_{M}^{H}(\pi)\right] / \varepsilon^{H}\right]
\end{aligned}
$$

where $\Delta=(1-\beta \pi)^{2} /\left[1-2 \beta \pi(1-\beta)-\beta^{2}\right]$. In addition, 


$$
\begin{aligned}
\bar{V}^{L}(0)= & {\left[\frac{1+\beta^{2} \pi-2 \beta \pi+\beta-\beta^{2}}{\left[1-\beta\left(\beta+\beta \pi^{2}+\pi-2 \beta \pi\right)\right](1-\beta \pi)}\right]\left[U\left(x^{L *}\right)-\left(x^{L *} / \varepsilon^{L}\right)\right]+} \\
& {\left[\frac{\beta(1-\pi)}{1-\beta\left(\beta+\beta \pi^{2}+\pi-2 \beta \pi\right)}\right]\left[U\left(x^{H *}\right)-\left(x^{H *} / \varepsilon^{H}\right)\right] } \\
\bar{V}^{H}(0)= & {\left[\frac{\beta(1-\pi)}{\left[1-\beta\left(\beta+\beta \pi^{2}+\pi-2 \beta \pi\right)\right](1-\beta \pi)}\right]\left[U\left(x^{L *}\right)-\left(x^{L *} / \varepsilon^{L}\right)\right]+} \\
& {\left[\frac{1}{1-\beta\left(\beta+\beta \pi^{2}+\pi-2 \beta \pi\right)}\right]\left[U\left(x^{H *}\right)-\left(x^{H *} / \varepsilon^{H}\right)\right] . }
\end{aligned}
$$

Suppose now $\varepsilon^{H}>\bar{\varepsilon}_{2}^{H}$ where $\bar{\varepsilon}_{2}^{H}$ is defined in Proposition 4. Define $\Phi^{L}(D ; \pi)=$ $W^{L}\left(z, D^{L} ; \pi\right)-\bar{W}^{L}\left(z, D^{L} ; \pi\right)+\left(1 / \varepsilon^{L}\right) D^{L}$. Notice that $\Phi^{L}\left(D^{L} ; \pi\right)$ is continuous on $D^{L} \in$ $\left(q^{L *}, q_{M}^{H}\right)$. Choose then $\delta>0$ so that $q^{L *}<\hat{D}^{L}-\delta<\hat{D}^{L}<\hat{D}^{L}+\delta<q_{M}^{H}$ where $\hat{D}^{L}$ comes from Proposition 2. As $\pi$ goes to $1, \varepsilon^{L} \Phi^{L}\left(D^{L} ; \pi\right)$ converges to $\varepsilon^{L} \Phi^{L}\left(D^{L}\right)$ on $\left[\hat{D}^{L}-\delta, \hat{D}^{L}+\delta\right]$. Then we can find $\bar{\pi}<1$ such that $\left|\varepsilon^{L} \Phi^{L}\left(D^{L} ; \pi\right)-\varepsilon^{L} \Phi^{L}\left(D^{L}\right)\right|<\delta$ on $\left[\hat{D}^{L}-\delta, \hat{D}^{L}+\delta\right]$ for $\pi \in(\bar{\pi}, 1)$. This implies the existence of a fixed point $\hat{D}^{L}(\pi)$, $\varepsilon^{L} \Phi^{L}\left(\hat{D}^{L} ; \pi\right)=\hat{D}^{L}(\pi)$ such that $q^{L *}<\hat{D}^{L}(\pi)<q_{M}^{H}(\pi)$ and hence a monetary equilibrium in which $L$-type buyers use credit only while $H$-type buyers use both money and credit. Here we use $q_{M}^{H}(\pi)>q_{M}^{H}$ for $\pi \approx 1$ because $\left[\partial q_{M}^{H}(\pi) / \partial \pi\right]<0$ for a sufficiently large $\varepsilon^{H}$ relative to $\varepsilon^{L}$ and $\lim _{\pi \rightarrow 1} q_{M}^{H}(\pi)=q_{M}^{H}$.

\section{References}

Araujo, Luis, and Tai-Wei Hu. (2014) "Optimal Monetary Interventions in Credit Markets." mimeo.

Berentsen, Aleksander, and Carlo Strub. (2009) "Central Bank Design with Heterogeneous Agents." European Economic Review, 53, 139-152.

Berentsen, Aleksander, Gabriele Camera, and Christopher Waller. (2007) "Money, Credit and Banking." Journal of Economic Theory, 135, 171-195.

Boel, Paola, and Gabriele Camera. (2009) "Financial Sophistication and the Distribution of the Welfare Cost of Inflation." Journal of Monetary Economics, 56, 968-978. 
Camera, Gabriele, and Yiting Li. (2008) "Another Example of a Credit System that Coexists with Money." Journal of Money, Credit and Banking, 40, 1295-1308.

Carapella, Francesca, and Stephen Williamson. (2015) "Credit Markets, Limited Commitment, and Government Debt." Review of Economic Studies, 82, 963-990.

Corbae, Dean, and Joseph Ritter. (2004) "Decentralized Credit and Public Exchange Without Public Record Keeping." Economic Theory, 24, 933-95140.

Cavalcanti, Ricardo, and Neil Wallace. (1999) "Inside and Outside Money as Alternative Media of Exchange." Journal of Money, Credit and Banking, 31, 443-457.

Gomis-Porqueras, Pedro, and Daniel Sanches. (2013) "Optimal Monetary Policy in a Model of Money and Credit." Journal of Money, Credit and Banking, 45, 701-730.

Gu, Chao, Fabrizio Mattesini, and Randall Wright. (2015) "Money and Credit Redux." Econometrica, In press.

Gu, Chao, and Randall Wright. (2015) "Monetary Mechanisms." mimeo.

Guerrieri, Veronica, Robert Shimer, and Randall Wright. (2010) "Adverse Selection in Competitive Search Equilibrium." Econometrica, 78, 1823-1862.

He, Ping, Lixin Huang, and Randall Wright. (2010) "Money, Banking and Monetary Policy." Journal of Monetary Economics, 55, 1013-1024.

Kahn, Charles M., and William Roberds. (2008) "Credit and Identity Theft." Journal of Monetary Economics, 55, 251-264.

Kiyotaki, Nobuhiro, and Randall Wright. (1989) "On Money as a Medium of Exchange." Journal of Political Economy, 97, 927-954.

Kiyotaki, Nobuhiro, and Randall Wright. (1993) "A Search-Theoretic Approach to Monetary Economics." American Economic Review, 83, 63-77.

Kocherlakota, Narayana (1998) "Money is Memory." Journal of Economic Theory, 81, 232-251.

Kocherlakota, Narayana, and Neil Wallace. (1998) "Incomplete Record-Keeping and Optimal Payment Arrangements." Journal of Economic Theory, 81, 272-289.

Lagos, Ricardo, Guillaume Rocheteau, and Randall Wright.(2015) "Liquidity: A New Monetarist Perspective." Journal of Economic Literature, forthcoming.

Lagos, Ricardo, and Randall Wright. (2005) "A Unified Framework for Monetary Theory and Policy Analysis." Journal of Political Economy, 113, 464-484.

Lotz, Sebastien, and Cathy Zhang. (2015) "Money and Credit as Means of Payment: A New Monetarist Approach." Journal of Economic Theory, In press. 
Nosal, Ed, and Guillaume Rocheteau. (2009) Money, Payments, and Liquidity. Cambridge: MIT Press.

Rocheteau, Guillaume, and Randall Wright. (2005) "Money in Search Equilibrium, in Competitive Equilibrium, and in Competitive Search Equilibrium." Econometrica, 73, 175-202.

Sanches, Daniel, and Stephen Williamson. (2010) "Money and Credit with Limited Commitment and Theft." Journal of Economic Theory, 145, 1525-1549.

Shi, Shouyong. (1996) "Credit and Money in a Search Model with Divisible Commodities." Review of Economic Studies, 63, 627-652.

Telyukova, Irina, and Randall Wright. (2008) "A Model of Money and Credit with an Application to the Credit Card Debt Puzzle." Review of Economic Studies, 75, 629-647.

Townsend, Robert M. (1989) "Currency and Credit in a Private Information Economy." Journal of Political Economy, 97, 1323-1344.

Wallace, Neil. (2010) "The Mechanism-Design Approach to Monetary Theory." Chapter 1 in Handbook of Monetary Economics, Volume 3A, edited by Benjamin Friedman and Michael Woodford, North Holland, 3-23.

Wallace, Neil. (2013) "An Attractive Monetary Model with Surprising Implications for Optima: Two Examples." mimeo, Pennsylvania State University. 